\title{
PAPEL DA CRIOCIRURGIA NO TRATAMENTO DAS NEOPLASIAS CUTÂNEAS DO SEGMENTO CABEÇA E PESCOÇO: ANÁLISE DE 1900 CASOS
}

\author{
ROLE OF CRYOSURGERY TREATMENT ON CUTANEOUS NEOPLASMS OF THE HEAD \\ AND NECK: ANALYSIS OF 1900 CASES
}

\author{
Antonio Azoubel Antunes ${ }^{1}$; Antonio Pessoa Antunes²; Polliana Vilaça Silva ${ }^{1}$
}

\begin{abstract}
RESUMO: Objetivo: Demonstrar a experiência dos autores na utilização da criocirurgia como tratamento de lesões benignas e malignas da pele e mucosa no segmento cabeça e pescoço. Método: Os autores realizam um estudo retrospectivo multicêntrico de 1900 casos de pacientes portadores de neoplasias benignas e malignas da cabeça e do pescoço, atendidos e tratados no Centro de Oncologia do Hospital Universitário Oswaldo Cruz (CEON/HUOC/UPE), Hospital de Câncer de Pernambuco (HCP) e clínica privada, no período de abril de 1977 a abril de 2002 (25 anos). Comparam, ainda, os dados obtidos com a revisão bibliográfica realizada, bem como sua experiência pessoal na utilização de tal modalidade terapêutica. Resultados: Do total de pacientes, $57,9 \%$ eram do sexo masculino e a quinta e sexta décadas de vida foram as mais frequentemente acometidas $(58,9 \%)$. O Carcinoma basocelular foi o tipo histológico predominante $(63,1 \%$ - 1200 casos), seguido dos hemangiomas (14,2\% - 270 casos). O tempo médio de exposição das lesões ao nitrogênio líquido foi de 15 e 35 segundos, e o tempo médio de cicatrização de 14 e 21 dias para as lesões benignas e malignas respectivamente. Conclusão: A indicação da criocirurgia deve obedecer alguns critérios de avaliação como o aspecto macroscópico e tamanho da lesão, tipo histológico, localização, idade e perfil social de cada paciente. Quando indicada e executada corretamente, oferece idênticos índices de cura aos outros métodos terapêuticos convencionais (Rev. Col. Bras. Cir. 2006; 33(2): 112-115).
\end{abstract}

Descritores: Criocirurgia; Crioterapia; Neoplasias de cabeça e pescoço.

\section{INTRODUÇÃO}

O método terapêutico cujo princípio básico consiste na agressão de lesões patológicas da pele e mucosas pelo congelamento intenso da área afetada, descreve-se sob a denominação de criocirurgia ${ }^{1}$.

$\mathrm{O}$ interesse da ação terapêutica do frio pela medicina remonta à antiguidade. Há 2.500 anos a.C. já havia informações nos Papiros egípcios que as feridas eram tratadas com o uso de compressas geladas, bem como as fraturas de crânio ${ }^{2}$.

Em 1889, Campbell White passou a usar o ar líquido especialmente em dermatologia, particularmente em forma de "spray". Porém foi Juliusberg, em 1903, quem iniciou o dióxido de carbono em forma de "spray" para o tratamento das lesões cutâneas, utilizado ainda hoje pelos especialistas ${ }^{3}$. Na década de 1940, o nitrogênio líquido assumiu a liderança entre as substâncias capazes de produzir baixas temperaturas, surgindo, desta forma, um novo campo na medicina, a criocirurgia.

Após o sucesso de alguns casos de criotalectomia no tratamento do Parkinsonismo, Cooper testou os efeitos do congelamento intenso na superfície de tumores, observando que a área congelada sofria necrose e esfacelo ocasionando o desaparecimento das células neoplásicas ${ }^{4}$.

Nos dias atuais, a criocirurgia encontra aplicação em quase todos os ramos da medicina, como na dermatologia, oftalmologia, proctologia, otorrinolaringologia, neurocirurgia, urologia, oncologia, e muitas outras especialidades.

Quando a moderna era da criocirurgia começou na década de 1960, a técnica era utilizada para tumores facilmente acessíveis à observação direta ou por via endoscópica, tais como pele, cavidade oral e próstata. Entretanto, na década de 1990, com o advento do ultra-som intra-operatório como método da monitorização do processo de congelamento e com o desenvolvimento de recursos criocirúrgicos mais efetivos, a utilização deste método para o tratamento dos tumores das vísceras e de outros tecidos profundos tornou-se prática usual ${ }^{5}$.

O objetivo do presente estudo é relatar a experiência dos autores com a referida modalidade terapêutica e discutir suas aplicações e aspectos de sucesso e insucesso no tratamento das neoplasias cutâneas da cabeça e do pescoço.

\section{MÉTODO}

Foi realizado um estudo retrospectivo, do período de Abril de 1977 a Abril de 2002, dos pacientes atendidos e tratados no Serviço de Cirurgia de Cabeça e Pescoço do Centro de Oncologia (CEON) do Hospital Universitário Oswaldo Cruz (HUOC) - Universidade de Pernambuco (UPE), e registrados no Centro Integrado de Anatomia Patológica

1. Acadêmico de Odontologia da FOP/UPE

2. Médico, Oncologista, Cirurgião de Cabeça e Pescoço.

Acadêmica de Odontologia da UFPE.

Recebido em 03/11/2005

Aceito para publicação em 29/12/2005

Conflito de interesses: nenhum

Fonte de financiamento: nenhuma

Trabalho realizado no Centro de Oncologia (CEON) do Hospital Universitário Oswaldo Cruz (HUOC) - Universidade de Pernambuco (UPE), Hospital de Câncer de Pernambuco (HCP) e Clínica Privada. 
Tabela 1 - Distribuição dos pacientes quanto à origem.

\begin{tabular}{lrl}
\hline & $\mathbf{N}$ & $\mathbf{\%}$ \\
\hline HCP/PE & 300 & 15,8 \\
CEON/HUOC/UPE & 1120 & 59,0 \\
Clínica privada & 480 & 25,2 \\
\hline
\end{tabular}

Tabela 2 - Distribuição dos pacientes por gênero.

\begin{tabular}{lrc}
\hline & $\mathbf{N}$ & $\mathbf{\%}$ \\
\hline Masculino & 1100 & 57,9 \\
Feminino & 800 & 42,1 \\
Total & $\mathbf{1 9 0 0}$ & $\mathbf{1 0 0 , 0 0}$ \\
\hline
\end{tabular}

Tabela 3 - Distribuição dos pacientes por faixa etária

\begin{tabular}{llr}
\hline & N & \% \\
\hline 00 a 20 anos & 120 & 6,3 \\
21 a 40 anos & 285 & 15,0 \\
41 a 60 anos & 1120 & 58,9 \\
61 a 80 anos & 310 & 16,3 \\
acima de 80 anos & 65 & 3,5 \\
Total & $\mathbf{1 9 0 0}$ & $\mathbf{1 0 0 , 0}$ \\
\hline
\end{tabular}

(CIAP), Hospital de Câncer de Pernambuco (HCP) e clínica privada.

Um total de 1900 pacientes portadores de neoplasias benignas e malignas das mais diversas localizações topográficas no segmento cabeça e pescoço foram selecionados. Pacientes com lesões irradiadas ou tratadas cirurgicamente sem sucesso foram incluídos no estudo. Entre os critérios de exclusão, consideramos os pacientes com lesões iguais ou maiores que $1,5 \mathrm{~cm}$ e lesões em outras localizações topográficas fora do segmento cabeça e pescoço. Todos os pacientes com lesões iguais ou maiores que $1 \mathrm{~cm}$ foram diagnosticados através de biópsia das lesões com pinça tipo "saca bocado", enquanto que as lesões menores receberam diagnóstico clínico.

A substância escolhida para o congelamento das lesões foi o nitrogênio líquido. No presente trabalho, foi utilizado o sistema aberto através do "Cryospray". O aparelho possui uma válvula para liberação do líquido no momento do uso, com pontas dermatológicas de diâmetros variáveis, com orifício central medindo entre 0,5 a $1,5 \mathrm{~mm}$. A ponta escolhida foi diretamente proporcional ao tamanho da lesão, localização topográfica e experiência pessoal do cirurgião. O tempo médio de exposição das lesões ao nitrogênio variou de 15 a 35 segundos para as lesões benignas e malignas respectivamente.

No pós-operatório, os pacientes foram orientados a realizar curativos diários nos primeiros sete dias, uso de analgésicos, antiinflamatórios, antialérgicos sistêmicos em casos específicos e antibioticoterapia tópica e/ou sistêmica quando necessário.
As variáveis estudadas foram o sexo, a idade, os tipos histológicos, e as características do tratamento e da cicatrização das lesões. Os tumores residuais foram definidos como lesões persistentes após o período de cicatrização, e foram todas diagnosticadas através de exame histopatológico das mesmas.

\section{RESULTADOS}

A Tabela 1 mostra a procedência dos pacientes. A maioria dos pacientes $(57,9 \%)$ foi do sexo masculino (Tabela $2)$, e esteve entre a quinta e sexta década de vida $(58,9 \%)$ (Tabela 3). Sessenta e três vírgula um por cento das lesões foram carcinomas basocelulares (CBC). Destes, 36,0\% eram menores que $1 \mathrm{~cm}$ e $27,1 \%$ mediam 1 e $1,5 \mathrm{~cm}$. O segundo tipo histológico mais frequentemente observado foram os hemangiomas $(14,2 \%)$ (Tabela 4).

A maioria dos pacientes com diagnóstico de $\mathrm{CBC}$ $(80,0 \%)$ não haviam se submetido a nenhuma forma de tratamento prévio das lesões (Tabela 5). Em média, as lesões malignas foram expostas ao nitrogênio líquido por um tempo mais prolongado, e seu período de cicatrização foi em média sete dias mais longo. As lesões residuais e recidivadas ocorreram unicamente nos CBCs. Todas as recidivas de casos de CBC envolveram pacientes com lesões iguais ou maiores que $1 \mathrm{~cm}$ de diâmetro e ocorreram entre 4 e 12 meses de acompanhamento.

Os 1200 casos de carcinomas basocelulares apresentaram as seguintes particularidades: (Tabela 5)

O tempo médio de exposição das lesões ao nitrogênio líquido foi de 15 segundos para as neoplasias benignas e de 35 segundos para as malignas.

O tempo médio de cicatrização das lesões benignas foi de 14 dias, enquanto que as malignas cicatrizaram em média de 21 dias.

O tumor residual esteve presente em 72 casos $(6,0 \%)$. A presença de recidiva tumoral foi identificada em 197 casos $(16,4 \%)$. Todos os casos de tumor residual e recidiva foram de carcinomas basocelulares.

\section{DISCUSSÃO}

A criocirurgia é um método terapêutico adotado para o tratamento de lesões benignas e malignas da pele e mucosas. É fundamentada em um processo físico que provoca morte celular pelo congelamento brusco e intenso, seguido do descongelamento lento, com provável ação na célula ou a na microcirculação.

No presente estudo, os autores demonstraram que a criocirurgia é um método terapêutico que pode ser amplamente utilizado no tratamento dos tumores superficiais benignos e malignos do segmento cabeça e pescoço, fornecendo resultados semelhantes aos métodos convencionais. Kuflik ${ }^{3}$, em estudo de 7338 casos de pacientes tratados pela criocirurgia durante um período de 30 anos, concluiu que a referida modalidade terapêutica é segura e efetiva para o tratamento do câncer de pele não-melanótico selecionado, com um índice de cura de aproximadamente $98,6 \%$. 
Tabela 4 - Distribuição dos pacientes por tipos histológicos.

\begin{tabular}{llrr}
\hline Tipo & Diagnóstico & N & \multicolumn{1}{c}{ \% } \\
\hline CBC: & Macroscópicos & 720 & 36,0 \\
& Microscópicos & 480 & 27,1 \\
Leucoplasias & & 180 & 9,5 \\
Hemangiomas & & 270 & 14,2 \\
Nevo pigmentado & & 100 & 5,2 \\
Hiperqueratoses & & 150 & 8,0 \\
Total & & $\mathbf{1 9 0 0}$ & $\mathbf{1 0 0 , 0}$ \\
\hline
\end{tabular}

Tabela 5 - CBC's - Particularidades.

\begin{tabular}{lrr}
\hline & $\mathbf{N}$ & $\mathbf{\%}$ \\
\hline Xeroderma pigmentosum & 18 & 1,0 \\
Irradiado com lesão residual & 50 & 2,6 \\
Irradiado e recidivado & 69 & 3,6 \\
Operado e recidivado & 60 & 3,1 \\
Tratado com quimioterapia tópica & 54 & 2,8 \\
Virgem de tratamento & 949 & 50,0 \\
Total & $\mathbf{1 2 0 0}$ & $\mathbf{6 3 , 1}$ \\
\hline
\end{tabular}

Flictenas são observadas 15 a 30 minutos após a aplicação da substância criogênica, dependendo da sensibilidade da pele do paciente, decorrente da agressão feita pelo nitrogênio. A mesma começa a adquirir um aspecto cianótico, observando-se tal fato com maior propriedade 24 horas após a aplicação do método terapêutico. A regeneração da epiderme inicia-se a partir do terceiro dia pós-aplicação, ocorrendo na epiderme que circunda a lesão um desprendimento lento e progressivo do tecido necrótico resultante. Naqueles casos em que ocorre infecção local da crosta necrótica, geralmente ocorre retardo da cicatrização. Tal fato foi claramente observado pelos autores.

O nitrogênio líquido, substância criogênica usada pelos autores no referido método terapêutico, é considerado a melhor substância pela maioria dos autores, pelas suas propriedades físicas a saber: ser inerte, inodoro, não combustível, não liberar gases tóxicos, mal condutor de calor e eletricidade e ter boa capacidade térmica ${ }^{5}$.

Waltz ${ }^{6}$ afirma em relação ao tempo de exposição da lesão ao nitrogênio líquido que após 30 segundos da aplicação, $80,0 \%$ da lesão já estará congelada, e para se conseguir o congelamento dos $20,0 \%$ restantes, a aplicação deverá estender-se por cerca de 5 minutos, afirmação esta com a qual não concordamos, por não ter observado tal fato em nosso material.

É importante registrar que a área de crionecrose é diretamente proporcional à dimensão da "bola de gelo" formada durante a criocirurgia. Deve-se, entretanto, observar durante a aplicação do método, os princípios básicos da cirurgia oncológica, ou seja, excisão da lesão com margens de ressecção cirúrgicas laterais e profundas satisfatórias, tomando o cuidado de congelar a lesão além do seu maior diâmetro evitando, desta forma, possíveis lesões residuais e/ou recidivas.
O aparecimento de uma área de despigmentação no local da lesão tratada pode ocorrer em pacientes melodermas pois enquanto os fibroblastos são muito resistentes à baixa temperatura as células normais, as terminações nervosas e os melanócitos são muito sensíveis, fato este que justifica tal despigmentação ${ }^{7}$.

Andrews ${ }^{8}$ refere como vantagens do tratamento um tempo curto de preparação, um baixo risco de infecção, a necessidade de cuidados mínimos com a ferida e o paciente não tem que retornar para retirada de pontos da ferida operatória. Dentre as principais vantagens do método terapêutico na experiência dos autores, registrou-se: simplicidade da técnica, economia de tempo para o paciente, não há necessidade de anestesia local, a possibilidade tratamento de várias lesões ao mesmo tempo, ocorrendo lesão residual o paciente poderá ser submetido ao tratamento cirúrgico convencional, boa cicatrização, pós-operatório não limita as atividades profissionais e sociais do paciente, tratamento seguro e pouco agressivo quando bem indicado, podendo ser efetuado em uma única sessão. Não observamos nos nossos pacientes, efeitos colaterais descritos por alguns autores, tais como neuropatias e fotosensibilidade ${ }^{9}$.

A literatura aponta como principais desvantagens: a dor, ardor e queimação durante o tratamento e a hipopigmentação local após descamação da crosta necrótica ${ }^{10}$. Outra limitação deste método são os resultados pobres com determinados tipos de lesão. Zhou et al ${ }^{7}$, comparando a criocirurgia feita em 107 pacientes portadores de melanoma maligno mucomembranoso da cavidade oral, com o tratamento convencional (cirurgia, quimioterapia e imunoterapia), concluem que o método terapêutico não deve ser utilizado para este tipo histológico de lesão pois a sobrevida dos pacientes tratados pela crioterapia foi de $0 \%$.

No presente estudo, os autores observaram a dor e queimação durante a aplicação, a impossibilidade de documentação histológica das pequenas lesões - o material necrótico eliminado não permite estudo histopatológico e a despigmentação no local da lesão após cicatrização total, particularmente em pacientes melanodermas como as principais desvantagens do método.

Um índice de cura de 100,0\% é referido com a utilização da criocirurgia como método terapêutico nos casos de carcinomas basocelulares iguais ou menores que $1,0 \mathrm{~cm}$ de diâmetro ${ }^{11}$, e o índice de recidiva global para estes tumores gira em torno de $10,0 \%{ }^{12}$. A recidiva tumoral ocorreu no carcinoma basocelular em 197 casos (16,4\%). Nenhum paciente com CBC menor que $1 \mathrm{~cm}$ de diâmetro apresentou recidiva tumoral, mostrando a importância da medida do diâmetro das lesões na escolha do método terapêutico. Todos os casos recidivados foram tratados com ressecção cirúrgica sem intercorrências.

\section{CONCLUSÕES}

1. A criocirurgia não deve ser adotada como método exclusivo no tratamento das lesões benignas e malignas da pele e mucosas, devendo sua indicação obedecer a alguns critérios de avaliação. Quando indicada e executada corretamente, oferece índices de cura semelhantes aos métodos convencionais. 


\begin{abstract}
Background: To demonstrate the authors' experience on cryosurgery treatment for malignant and benign skin neoplasms on head, neck including mucosa. Methods: The authors review a multicentric retrospective study of 1900 cases of patients with benign and malignant head and neck neoplasms attended and treated at CEON/HUOC/UPE, HCP and private clinic, from April 1977 to April 2002 (25 years). They also compare the data obtained with the literature review, and show their personal experience on its use. Results: Within all cases registered, $57.9 \%$ were male and the fifth and sixth decades of life were most common (58.9\%). The most prevalent histologica type was basal cell carcinoma (63.1\% - 1200 cases), followed by hemangiomas (14.2\% - 270 cases). The mean time of lesions exposure to liquid nitrogen was 15 and 35 seconds and the mean cicatrisation time were 14 and 21 days, for benign and malignant lesions, respectively. Conclusion: Cryosurgery indication must obey a few evaluation criteria, such as macroscopic aspect and lesion size, histological type, location, age and social profile. When correctly indicated and executed, it obtains identical cure rates as other conventional therapeutic methods.
\end{abstract}

Key words: Cryosurgery; Cryotherapy; Head and neck neoplasms.

\section{REFERÊNCIAS}

1. Barbosa JF, Sanvitto LC. Crioterapia local (Criocirurgia) - denominação e histórico. Bol Oncol. 1973; 63(3-4):29-34.

2. Miller T. Cryosurgery. Aula no "Cursus de Atualisacion en Diagnostico y terapêutica del cancer". Congresos Integrados Latinamericanos de Cancerologia. Caracas. Venezuela, 10 a 15 de outubro de 1971.

3. Kuflik EG. Cryosurgery for skin cancer: 30-year experience and cure rates. Dermatol Surg. 2004; 30(2 Pt 2):297-300.

4. Barbosa JF, Sanvitto LC. Crioterapia local (Criocirurgia) - Mecanismos da ação do brusco e intenso resfriamento. Bol Oncol. 1973; 63(3-4):35-44.

5. Gage AA, Baust JG. Cryosurgery for tumors - a clinical overview. Technol Cancer Res Treat. 2004; 3(2):187-99.

6. Waltz JM, Cooper JS. Cryosurgery of brain lesions. J St Barnabas Med Center. 1967; 4:274-84.

7. Zhou GY, Zhang ZY, Lin GC, Gu JZ, Qiu WL. [Evaluation of cryosurgery for the management of oral malignant mucomembranous melanoma - A survey of 107 cases reports]. Shangai Kou Qiang Yi Xue. 1999; 8(2):92-3.

8. Andrews MD. Cryosurgery for common skin conditions. Am Fam Physician. 2004; 69(10):2365-72.

9. Wooldridge JS. Treatment of skin cancer by cryosurgery. Missouri Med. 1975; 28-34.
10. Thai KE, Fergin P, Freeman M, Vinciullo C, Francis D, Spelman L, Murrell D, Anderson C, Weightman W, Reid C, Watson A, Foley P. A prospective study of the use of cryosurgery for the treatment of actinic keratoses. Int J Dermatol. 2004; 43(9)68792.

11. Giuffrida TJ, Jimenez G, Nouri K. Histologic cure of basal cell carcinoma treated with cryosurgery. J Am Acad Dermatol. 2003; 49(3):483-6.

12. Kokoszka A, Scheinfeld N. Evidence-based review of the use of cryosurgery in treatment of basal cell carcinoma. Dermatol Surg. 2003; 29(6):556-71.

Como citar este artigo:

Antunes AA, Antunes AP, Silva PV. Papel da criocirurgia no tratamento das neoplasias cutâneas do segmento cabeça e pescoço: análise de 1900 casos. Rev Col Bras Cir. [periódico na Internet] 2006 Mar-Apr; 33(2). Disponível em URL: http://www.scielo.br/rcbc

Endereço para correspondência:

Antonio Azoubel Antunes

Rua Guilherme Pinto, 345/303

Derby

52010-210 - Recife - PE

E-mail: antunesctbmf@yahoo.com.br 